\title{
PAPER
}

\section{Toxins and the gut: role in human disease}

\section{A Fasano}

Bacterial enteric infections exact a heavy toll on the human population, particularly among children. Despite the explosion of knowledge on the pathogenesis of enteric diseases experienced during the past decade, the number of diarrhoeal episodes and human deaths reported worldwide remains of apocalyptic dimensions. However, our better understanding of the pathogenic mechanisms involved in the onset of diarrhoea is finally leading to preventive interventions, such as the development of enteric vaccines, that may have a significant impact on the magnitude of this human plague. The application of a multidisciplinary approach to study bacterial pathogenesis, along with the recent sequencing of entire microbial genomes, have made possible discoveries that are changing the way scientists view the bacterium-host interaction. Today, research on the molecular basis of the pathogenesis of infective diarrhoeal diseases of necessity transcends established boundaries between microbiology, cell biology, intestinal pathophysiology, and immunology. This review focuses on the most recent outcomes of this multidisciplinary effort.

Correspondence to: Dr A Fasano, Division of Pediatric Gastroenterology and Nutrition, University of Maryland School of Medicine, $685 \mathrm{~W}$ Baltimore St HSF Building, Room 465, Baltimore, MD 21201, USA;

afasano@umaryland.edu
M icroorganisms represent the first species of living organisms that populated our planet and will probably continue to survive well beyond the extinction of the human race. Their distinguishing characteristics (small size, concise deployment of genetic information, and ability to survive in highly varied circumstances) contribute to their acclaimed virtuosity to adapt and to learn fast in order to survive. To be a successful enteric pathogen, a microorganism has to be a good coloniser, compete for nutrients, and to be able to interact with the target eukaryotic cell in order to induce secretion of water and electrolytes. As the basic metabolism of enteric pathogens and commensals is the same, it follows that pathogens must possess highly specialised attributes which enable them to activate one of the eukaryotic intracellular pathways leading to intestinal secretion (for a comprehensive review, see Fasano ${ }^{1}$ ). This cross talk between enteric pathogens and intestinal host may be activated by either invasion or elaboration of toxins. This review will focus on this last mechanism of action by reviewing recent reports on toxins elaborated by the most common enteric pathogens (table 1).

\section{TOXINS THAT ACTIVATE ENTEROCYTE SIGNAL PATHWAYS}

Intestinal cells operate through three well established intracellular signal transduction pathways to regulate water and electrolyte fluxes across the intestinal mucosa: cyclic adenosine monophosphate (cAMP); cyclic guanosine monophosphate (cGMP); and calcium dependent pathways (fig 1). Recently, a fourth pathway involving nitric oxide (NO) has been described.

\section{CAMP}

An extremely heterogeneous group of microorganisms, Escherichia coli encompass almost all features of possible interaction between intestinal microflora and the host, ranging from a role of mere harmless presence to that of highly pathogenic organisms. In fact, the $E$ coli species is made up of many strains that profoundly differ from each other in terms of biological characteristics and virulence properties. ${ }^{2}$ Among the pathogenic $E$ coli, enterotoxigenic $E$ coli (ETEC) represent the archetype of toxin producing $E$ coli. The heat labile toxin (LT) produced by some ETEC strains is structurally and functionally similar to cholera toxin (CT) produced by Vibrio cholerae, and both activate the adenylate cyclase/cAMP pathway. ${ }^{1}$ However, while LT induces a mild diarrhoea known as "travellers" diarrhoea, CT is responsible for the severe, sometimes fatal, clinical condition typical of cholera. CT and LT share a common structure consisting of an A (active) subunit of $\sim 25 \mathrm{kDa}$ and a ring of $5 \mathrm{~B}$ (binding) subunits of $\sim 11 \mathrm{kDa}$ each (AB5). Recently, Rodighiero et al have reported that the differential toxicity of CT and LT is related to a 10 amino acid segment within the A2 fragment of CT that confers a higher stability to the CT holotoxin during uptake and transport into intestinal epithelia. ${ }^{3}$

\section{cGMP}

Besides LT, ETEC elaborate a family of heat stable enterotoxins (STs). STIp is a small peptide that stimulates guanylate cyclase, causing an increased intracellular concentration of CGMP, which evokes chloride secretion and diarrhoea. ${ }^{1}$ STIp is a typical extracellular toxin consisting of 18 amino acid residues synthesised as a precursor protein. The precursor translocates across the

Abbreviations: $A N P$, atrial natriuretic peptide; $B F T, B$ fragilis enterotoxin; $C P E, C$ perfringens enterotoxin; $C T$, cholera toxin; CTC, V cholerae cytolysin; EAggEC, enteroaggregative $E$ coli; ETEC, enterotoxigenic $E$ coli; LPS, lipopolysaccharide; GC, guanylate cyclase; LT, heat labile toxin; PET, plasmid encoded protein; PKC, protein kinase C; ShET, Shigella enterotoxin; ST, heat stable toxin; $\mathrm{TDH}$, thermostable direct haemolysin 


\author{
Table 1 Enteric toxins \\ Toxins that activate enterocyte signal pathways \\ Cyclic AMP \\ Cholera toxin (CT) \\ Heat labile Escherichia coli enterotoxin (LT) \\ Salmonella enterotoxin \\ Campylobacter jejuni enterotoxin \\ Pseudomonas aeruginosa enterotoxin \\ Shigella dysenteriae enterotoxin \\ Cyclic GMP \\ Heat stable Escherichia coli enterotoxin (ST) \\ Yersinia enterocolitica ST I and ST II enterotoxins \\ Yersinia bercovieri enterotoxin \\ Klebsiella pneumoniae enterotoxin \\ Heat stable Vibrio cholerae non-O1 enterotoxin \\ Enteroaggregative Escherichia coli heat stable enterotoxin (EAST1) \\ Calcium \\ Clostridium difficile enterotoxin \\ Ciguatera enterotoxin \\ Cryptosporidium enterotoxin \\ Helicobacter pylori vacuolating toxin \\ Vibrio parahaemolyticus thermostable direct haemolysin (TDH) \\ Nitric oxide \\ Shigella flexneri 2a Shigella enterotoxin 1 (ShET1) \\ Pore forming toxins \\ Clostridium perfrigens enterotoxin (CPE) \\ Staphylococcus aureus $\alpha$ toxin \\ Vibrio cholerae cytolysin (CTC) \\ Toxins blocking protein synthesis \\ Shigella dysenteriae Shiga toxin \\ EHEC Shiga like toxin 1 (SLT 1) and 2 (SLT 2) \\ Toxin inducing protein synthesis \\ Staphylococcus aureus enterotoxin A \\ EAggEC toxin \\ Toxins affecting the enterocyte cytoskeleton \\ Clostridium difficile toxin $A$ and $B$ \\ Clostridium sordelli toxin \\ Clostridium botulinum $\mathrm{C} 2$ and $\mathrm{C} 3$ toxins \\ Escherichia coli cytotoxic necrotising factor 1 (CNF 1) \\ Campylobacter jejuni cytolethal distending toxin \\ Vibrio cholerae Zonula occludens toxin (Zot) \\ EAggEC plasmid encoded protein (PET) \\ Bacteroides fragilis toxin (BFT) \\ Vibrio parahaemolyticus thermostable direct haemolysin (TDH)
}

inner membrane utilising the general export pathway consisting of the Sec proteins. ${ }^{4}$ TolC, an ETEC outer membrane protein, seems to be necessary for the translocation of the toxin across the outer membrane, as deletion mutants of the tolC gene release less STIp into the culture supernatant and do not induce secretion in vivo in the mouse ileal loop model. ${ }^{5}$ In addition to LT and ST exotoxins, ETEC also contain a lipopolysaccharide (LPS) endotoxin. When orally administered to mice, LPS greatly increased the expression of the inducible nitric oxide synthase II (NOS II) and its effector enzyme soluble guanylate cyclase in colonic cells. ${ }^{6}$ This creates the pathophysiological autocrine pathway producing increased levels of cGMP and leading to hypersecretion and diarrhoea. ${ }^{6}$ Another heat stable enterotoxin (EAST1) genetically and structurally distinct from ST was originally discovered in enteroaggregative $E$ coli (EaggEC $)^{7}$ and subsequently found in other $E$ coli belonging to several distinct diarrhoeogenic categories. ${ }^{8} \mathrm{~A}$ recent case-control study showed that $19 \%$ of children with diarrhoea harboured EASTl positive $E$ coli in their stools compared to $3.5 \%$ isolated from healthy individuals, ${ }^{9}$ confirming the pathogenic role of EASTl in diarrhoeal diseases in children. The last described member of the ST family has been recently reported by Sulakvelidze and coworkers. ${ }^{10}$ This toxin elaborated by Yersinia bercovieri elicited a secretory response in both in vitro and in vivo animal models; however, it was genetically and immunologically distinct from Yersinia enterocolitica ST I, ST II, and other known enterotoxins. ${ }^{10}$

\section{Calcium}

Several toxins, including ciguatera toxin, ${ }^{11}$ Clostridium difficile toxin, ${ }^{12}$ Cryptosporidium toxin, ${ }^{13}$ and the Helicobacter pylori vacuolating toxin ${ }^{14}$ seem to act through Ca. However, the involvement of $\mathrm{Ca}$ in the secretory effect of these toxins has been only indirectly shown. A more definitive proof of the Ca mediated secretory effect has been provided by Raimondi et al, who have shown, using direct $[\mathrm{Ca}]_{\text {, }}$ measurement, that the enterotoxic effect of the thermostable direct haemolysin (TDH) elaborated by Vibrio parahaemolyticus is mediated by $\mathrm{Ca}^{15}$ This toxin seems to interact with a polysialoganglioside GTlb surface receptor, whose physiological function remains to be established. ${ }^{15}$

\section{Nitric oxide}

The role of NO in intestinal fluid and electrolyte balance varies according to the pathophysiological conditions that activate this pathway. Under physiological circumstances, NO exerts a proabsorptive effect that involves the enteric nervous system. ${ }^{16}$ However, high NO production has been shown in both animal models $\mathrm{s}^{16-19}$ and humans ${ }^{20}$ to contribute to diarrhoea by acting as a secretagogue.

Our laboratory has described the elaboration by Shigella flexneri 2a of two novel iron regulated enterotoxins, named Shigella enterotoxin 1 (ShET1) and 2 (ShET2), that alter electrolyte and water transport in rabbit small intestine both in vitro and in vivo. $^{21}{ }^{22} \mathrm{ShETl}$ is a chromosomally encoded, $55 \mathrm{kDa}$ complex protein $^{21}$ that is universally elaborated by Shigella flexneri 2a strains but only rarely by other serotypes. ${ }^{23}$ ShET 1 appears to exert an irreversible, dose dependent enterotoxic effect that does not seem to be mediated by Ca, cAMP, or cGMP. Recent studies have shown that, when tested on rabbit, rat, and mouse intestines in vitro, ShETl induces an increased $\mathrm{NO}_{2}^{-}$concentration in the bathing solution that is partially blocked by the coadministration of inducible NO synthase (iNOS) inhibitors. ${ }^{24}$ The direct effect of the toxin on iNOS expression has been confirmed by RT-PCR, Northern blot analysis, and by experiments conducted on a iNOS knock out mouse model. ${ }^{24}$

\section{PORE FORMING TOXINS}

Clostridium perfrigens is a common agent of food borne intoxication whose symptoms are caused by the elaboration of $C$ perfrigens enterotoxin (CPE) (reviewed in Popoff ${ }^{25}$ ). CPE is a very hydrophobic protein that is released by bacterial lysis and subsequently binds to a brush border receptor of the host enterocyte. ${ }^{25}$ Following this binding, CPE associates with a 70 $\mathrm{kDa}$ membrane protein with subsequent formation of pores through which water, ions, nucleotides, and amino acids leak. Staphylococcus aureus $\alpha$ toxin also forms pores; however, its mechanism of action involves the formation of oligomers containing only toxin molecules. ${ }^{25}$ According to Zitzer and coworker, the Vibrio cholerae cytolysin (CTC) represents a novel prototype of pore forming toxin. ${ }^{26}$ The authors have recently shown that the oligemirasation of CTC yields a pentameric pore and has a dual specificity for both cholesterol and ceramides present in the mammalian brush border membrane of enterocytes. ${ }^{26}$

\section{TOXINS BLOCKING PROTEIN SYNTHESIS}

Shiga toxin elaborated by Shigella dysenteriae represents the archetype of this family of toxins. Shiga-like toxins (SLT) 1 and 2 are related toxins elaborated by enterohaemorrhagic $E$ coli (EHEC), a microorganism implicated in haemorrhagic colitis and haemolytic uraemic syndrome. ${ }^{27}$ Shiga toxin and SLTs share the AB5 structure typical of CT and LT, however they act through a different mechanism of action. The Al subunit of Shiga toxin and SLTs binds and inactivates the 60S subunit of the host cell ribosome and, consequently, completely interrupts the cell protein synthesis. ${ }^{25}$ In order to induce this 


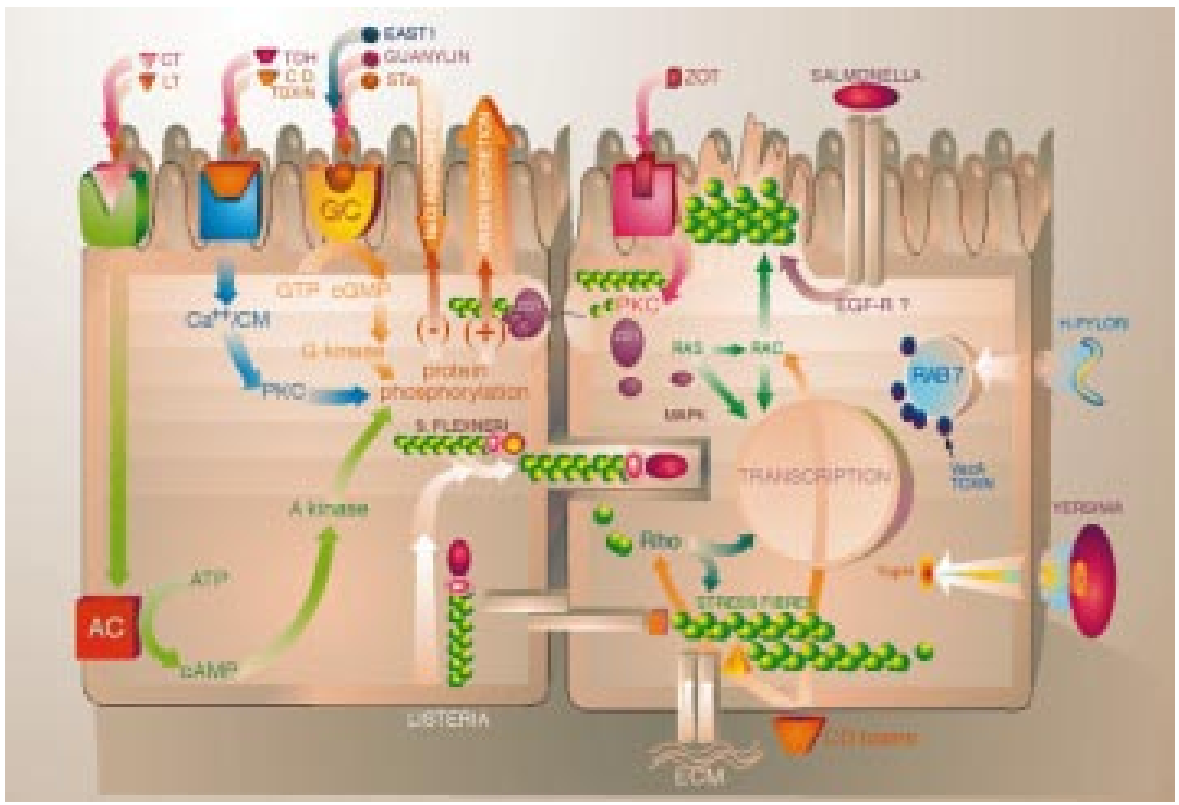

Figure 1 Enterocyte intracellular signalling leading to intestinal secretion. Four main pathways seems to be involved in the intestinal secretion of water and electrolytes: $c A M P, C G M P, C a$, and cytoskeleton. These pathways are activated by several enteric pathogens, either directly or through the elaboration of enterotoxic products. CT, cholera toxin; LT, heat labile enterotoxin; TDH, thermostable direct hemolysin; C.D., Clostridium difficile; EAST1, enteroaggregative $E$ coli heat stable toxin 1; STa, heat stable toxin a; $A C$, adenylate cyclase; $G C$, guanylate $c y c l a s e ; ~ C M$, calmodulin; PKC, protein kinase C; ZOT, Zonula occludens toxin; EGF-r, epidermal growth factor receptor; ECM, extracellular matrix.

inhibitory effect, the toxins must interact with a glycolipid surface receptor ( $\mathrm{Gb} 3$ receptor) whose expression in different endothelial domains varies. ${ }^{27}$ In fact, while endothelial cells of large blood vessels, such as umbilical and saphenous veins, produce minimal amounts of $\mathrm{Gb}^{2}{ }^{28}$ human renall ${ }^{28}$ and intestinal $^{29}$ microvascular endothelial cells constitutively express maximal quantities of the receptor. These results provide a rationale for the targeting of the glomeruli in haemolytic uraemic syndrome and the endothelial cells of the colon in haemorrhagic colitis. Recent epidemiological data suggest that the elaboration of SLTs by itself may not be sufficient to induce disease in humans. By applying a multivariate logistic regression analysis, Boerlin and coworkers showed a significant association between the presence of genes for intimin (a protein involved in the intimate attachment of EHEC to the host intestinal cell) and SLT2 and isolates from cases of haemorragic colitis and haemolytic uraemic syndrome. ${ }^{30}$ Further analysis revealed an interaction between the intimin gene and the SLT2 gene, supporting the hypothesis of the synergism between the adhesin intimin and SLT2. ${ }^{30}$

\section{TOXINS INDUCING PROTEIN SYNTHESIS}

Up regulation of protein synthesis, particularly proinflammatory mediators, is one of the most recently described mechanisms through which bacterial toxins induce diarrhoea. Nielsen and coworkers have shown that staphylococcal enterotoxin A induces tyrosine phosphorylation of several host intracellular proteins, down regulation of the $\mathrm{T}$ cell receptor, and production of interferon $\gamma$, a key cytokine in the pathogenesis of intestinal inflammatory and secretory processes. ${ }^{31}$ Transcriptional up regulation of proinflammatory cytokines seems also to be involved in the pathogenesis of EaggEC associated diarrhoea. ${ }^{32}$ It has been recently reported that EAggEC produce a cell free factor that up regulates interleukin 8 (IL-8) messenger RNA in CaCo2 cells. ${ }^{33}$ This up regulation correlates with the clinical observation that increased lactoferrin (as a marker of inflammation) and IL-8 can be found in stools of children in Brazil with EAggEC infections. ${ }^{33}$

\section{TOXINS AFFECTING THE ENTEROCYTE ACTIN CYTOSKELETON}

A growing number of toxins have been reported to act by affecting the host cell cytoskeleton. Clostridium difficile has emerged as the most important pathogen causing the syndrome of antibiotic associated colitis. ${ }^{34} \mathrm{C}$ difficile infections range in severity from asymptomatic forms to clinical syndromes such as severe diarrhoea, pseudomembranous colitis, toxic megacolon, and even death. ${ }^{34}$ The virulence of this pathogen is dependent on its elaboration of two related toxins TxA and TxB. These toxins are among the largest monomeric toxins described, with molecular weights of 308000 for TxA and 270000 for TxB. Despite the fact that TxA has traditionally been referred to as an enterotoxin and $T \times B$ as a cytotoxin, ${ }^{34}$ they both exert a cytotoxic effect in vitro. Both TxA and $\mathrm{TxB}$ are glucosyltransferases and use UDP glucose as a substrate to inactivate by monoglucosylation members of the Rho family of small GTPases at $\mathrm{Thr}^{37}$, an amino acid residue located within the putative effector domain of the Rho proteins. $^{35}$ Rho GTPases regulate a variety of cytoskeleton dependent cellular functions such as cell adhesion and motility, growth factor mediated signalling, cellular transformation, and induction of apoptosis. ${ }^{36}$ The dramatic effects of TxA and TxB on tissues and cells, including cytoskeletal depolymerisation, increased intestinal permeability and diarrhoea, cellular retraction and rounding, disruption of cell adhesion and chemotaxis, and activation of apoptosis, ${ }^{37}$ are therefore all related to the TxA and TxB dependent inactivation of the Rho proteins. Clostridium sordelli toxin also functions as a UDP glucosyl transferase and inactivates Ras, Rap, and Rac. ${ }^{32}$ Clostridium botulinum C2 and C3 toxins exert their enterotoxic effect by inactivating actin and Rho, respectively. ${ }^{32}$

Beside the inactivation of Rho proteins, their activation is also associated with increased intestinal permeability and diarrhoea. Cytotoxic necrotising factor l (CNF1), an $\sim 115 \mathrm{kDa}$ protein produced by pathogenic Escherichia coli strains, ${ }^{38}$ activates Rho GTPases by deamination of $\mathrm{Gln}^{63}$, and consequently induces polymerisation of $\mathrm{F}$ actin. ${ }^{39}$ When tested on Caco2 monolayers, CNF1 reduced the monolayer resistance by $40 \%$ after four hours of incubation, ${ }^{40}$ suggesting that not only 


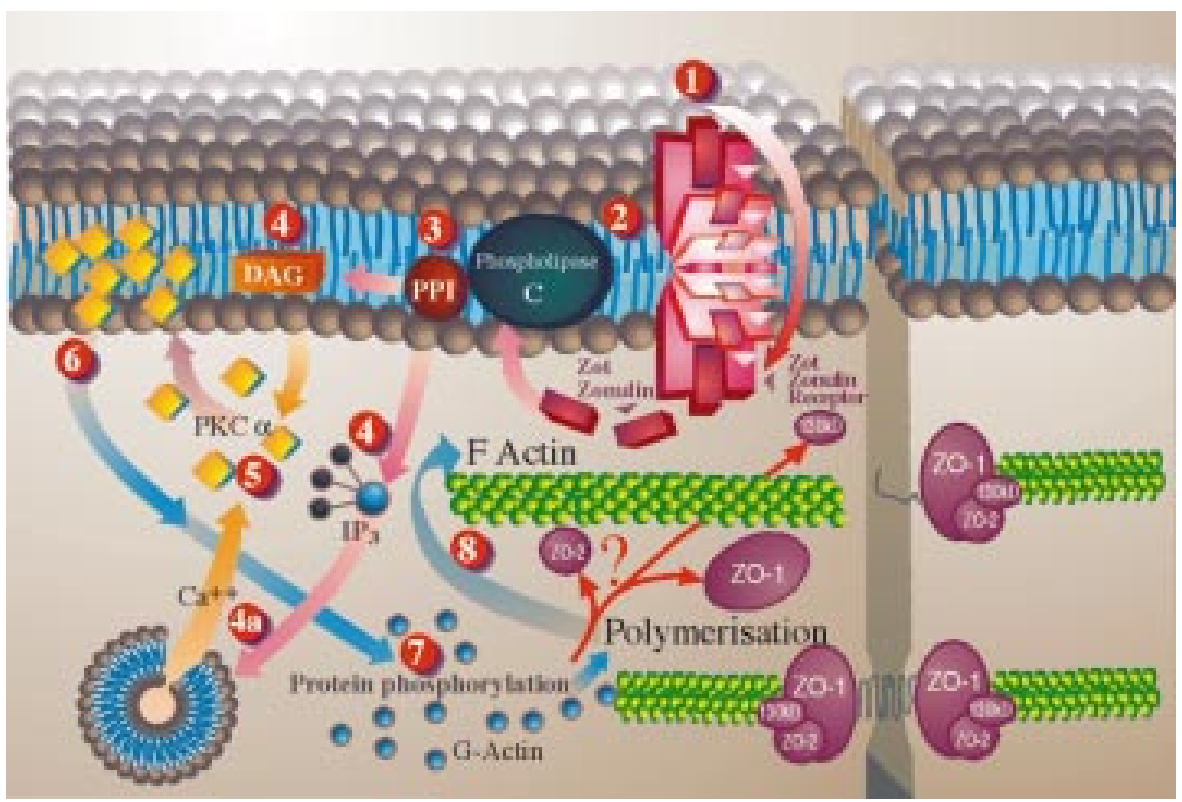

Figure 2 Proposed Zot/zonulin intracellular signalling leading to the opening of intestinal tight junctions. The molecules interact with a specific surface receptor (1) whose distribution within the intestine varies. The proteins are then internalised and activate phospholipase C (2) which hydrolyses phosphatidyl inositol (3) to release inositol 1,4,5-tris phosphate (IP3) and diacylglycerol (DAG) (4). PKC $\alpha$ (5) is then activated (6), either directly (via DAG) (4) or through the release of intracellular $\mathrm{Ca}^{++}$(via IP3) (4a). PKC $\alpha$ catalyses the phosphorylation of target protein(s) with subsequent polymerisation of soluble $G$ actin in $F$ actin (7). This polymerisation causes the rearrangement of the filaments of actin and the subsequent displacement of proteins (including ZO1) from the junctional complex (8). As a result, intestinal tight junctions become looser.

depolymerisation but also polymerisation of actin and subsequent reorganisation of the actin cytoskeleton alter the barrier function of intestinal tight junctions.

A similar mechanism was previously described for Zonula occludens toxin (Zot), a toxin elaborated by Vibrio cholerae. ${ }^{41} 42$ Zot is a single polypeptide chain of $44.8 \mathrm{kDa}$ encoded by the bacteriophage СТХ $\Phi$ present in toxigenic strains of Vibrio cholerae ${ }^{43}$ Zot increases the intestinal permeability by interacting with a mammalian cell receptor, with subsequent activation of an intracellular signalling, leading to the disassembly of the intercellular tight junctions (fig 2) ${ }^{42}{ }^{44}$ Zot localises in the bacterial outer membrane of $V$ cholerae with subsequent cleavage and secretion of a $\mathrm{C}$ terminal fragment in the host intestinal milieu. ${ }^{45}$ Structure-function analysis of the toxin suggests that Zot has a dual function: while its $\sim 33 \mathrm{kDa} \mathrm{N}$ terminal portion is possibly involved in the CTX $\Phi$ phage assembly, the $\sim 12 \mathrm{kDa} C$ terminal fragment of the toxin seems responsible for the permeating action on intestinal tight junctions ${ }^{46}$ Interestingly, the Zot $\mathrm{C}$ terminal fragment shares a putative receptor binding motif with zonulin, the recently described Zot mammalian analogue involved in tight junction modulation. ${ }^{47}$ Amino acid comparison between the Zot active fragment and zonulin, combined with site directed mutagenesis experiments, confirmed the presence of an octapeptide receptor binding domain towards the $\mathrm{N}$ terminus of the processed Zot. ${ }^{47}$

The plasmid encoded protein (PET) elaborated by EAggEC is a member of the autotransporter class of secreted proteins that induces contraction of the cytoskeleton and loss of the actin stress fibres when tested on either Hep-2 cells or HT29 C cell monolayers. ${ }^{48}$ The cytopathic and enterotoxic effects of PET seem to be related to the serine protease activity of the toxin that elicits cytoskeletal changes without compromising cell viability. ${ }^{48}$

Enterotoxigenic Bacteroides fragilis elaborate a $20 \mathrm{kDa}$ zinc dependent metalloprotease toxin (Bacteroides fragilis enterotoxin, BFT) that alters tight junctions and intestinal permeability. ${ }^{49}$ BFT specifically cleaves the extracellular domain of the zonula adherens protein E cadherin. BFT protease activity appears to be specific for E cadherin, as no proteolytic activity was detected for other cytoskeletal associated proteins, including occludin, $\beta_{1}$ integrin, ZOl, or $\alpha$ and $\beta$ catenins. ${ }^{49}$
In addition to its Ca mediated enterotoxic effect mentioned above, the Vibrio parahaemolyticus enterotoxin TDH also induces a significant, though reversible, decreased rate of progression through the cell cycle and morphological changes related to the organisation of the microtubular network, which appears to be the preferential cytoskeletal element involved in the cellular response to the toxin. ${ }^{50}$

\section{HOW TOXIN GENES ARE ACQUIRED?}

Virulence genes of pathogenic bacteria, which code for toxins that act through one of the pathways outlined above, are acquired via transmissible genetic elements such as transposons, plasmids, or bacteriophages. In addition, such genes may be part of particular regions on the bacterial chromosome termed "pathogenicity islands". ${ }^{1}$ These islands contain DNA sequences for bacteriophage attachment, suggesting that the genes present within the pathogenicity islands were previously able to spread among bacterial populations by horizontal gene transfer (via phage transfection), a process known to contribute to microbial evolution. This phenomenon has been elegantly shown to occur in Vibrio cholerae by Waldor and Mekalanos. ${ }^{43}$ The authors have shown that the genes upstream ct $x$ belong to a filamentous phage (designed CTX $\phi$ ) that replicates as a plasmid and is responsible for the horizontal transfer of a pathogenic element $(c t x)$ to non-toxigenic Vibrio cholerae. ${ }^{43}$ Analysis of clinical and environmental nontoxigenic Vibrio cholerae strains revealed that they can be infected by the phage either in vitro or in the intestines of infant mice. ${ }^{51}$ The phage genome integrated into the chromosome of infected Vibrio cholerae Ol cells forming stable lysogens, suggesting that lysogenic conversion of nontoxigenic Vibrio cholerae by CTX $\phi$ can naturally occur, leading to the origination of potential new endemic clones. This hypothesis has been recently confirmed by Kimsey and coworkers ${ }^{52}$ with the demonstration that the new outbreak of cholera which occurred in Calcutta in 1996 was caused by a Vibrio cholera O139 that acquired a CTX $\phi$ distinct from that present in the Vibrio cholerae O139 in Bengal in 1992.

A revolutionary mechanism of acquisition of bacterial pathogenicity has recently been reported by Maurelli and 
coworkers. $^{53}$ The authors showed for the first time that virulence traits may be acquired not only with the acquisition of virulence genes but also with the shedding of genes that are detrimental to new pathogenic lifestyles. Their results showed that, as Shigella spp evolved from $E$ coli to become pathogens, they not only acquired virulence genes on a plasmid but also lost genes via deletion. The formation of these "black holes", deletion of genes that exert an inhibitory effect on toxins' functions, provide an evolutionary pathway that enables a pathogen to enhance virulence.

\section{ENTEROTOXINS MIMICKING EUKARYOTIC COUNTERPARTS}

Infectious diseases have been traditionally perceived as a human plague that needs to be aggressively fought in order to get rid of the harmful microorganisms. However, to bacteria, illness is often inadvertent, the result of subtle subversion of the eukaryotic cell functions exploited by microorganisms to achieve their own profit. In this learning process, enteric pathogens have selected their strategies to mimic eukaryotic cell signalling factors and, therefore, innovative ways to finely "tune" the activity of the host cell regulatory pathways. This knowledge is contributing to our understanding of the complex host-bacteria relation and may lead to the development of innovative strategies for the treatment and prevention of human diseases.

Because of the magnitude and dynamism of this new field of research, it is impossible to cover all the areas of current research in a single review. Therefore, this paper has focused on two representative examples of what is presently known about the interaction between enteric pathogens and the host cell, with most of the emphasis on the author's personal research experience.

\section{The guanylin system}

The ST epithelial surface receptor is distinct from the CT and LT toxin receptors and coincides with guanylate cyclase (GC) activity. ${ }^{54}{ }^{55}$ Ileal villous epithelial cells have approximately twice as many receptors as crypt cells for the enterotoxin. ${ }^{56} \mathrm{GC}$ exists in two major forms, soluble and particulate. These are distinct proteins encoded by separate genes. Soluble GC is a dimeric cytosolic protein that is activated by nitric oxide. ${ }^{57}$ Particulate GC is a family of brush border membrane glycoproteins that are activated by only two classes of substances, atrial natriuretic peptides (ANPs) and ST. In the intestine, approximately $80 \%$ of total guanylate cyclase is particulate. So far, three different members of the particulate GC family have been cloned. ${ }^{58}{ }^{59} \mathrm{GC}-\mathrm{A}$ and GC-B are ANP receptor cyclases, while GC-C is the specific receptor for ST. The physiological role of the ST receptor in the mammalian intestine was unknown until a few years ago, when Currie and coworkers ${ }^{60}$ extracted and purified from the rat small intestine a peptide that is homologous to ST. This endogenous peptide, named guanylin, has been shown to be $50 \%$ homologous to ST and to bind competitively to the ST binding site on T84 cells, thereby stimulating cyclic GMP production (table 1). ${ }^{60}$ Our group has described a heat stable enterotoxin (named EAST1) elaborated by EaggEC ${ }^{61}$ that proved to be structurally and functionally similar to guanylin. ${ }^{7}$ Studies on T84 cells and COS cells tranfected with GC-C suggest that EASTl interacts with GC-C to elicit an increase in cyclic GMP (D Robertson, A Fasano, S Savarino, personal communication). The EASTl genotype is not restricted to EaggEC, being detected with notable frequency in enterohaemorrhagic $E$ coli (EHEC), ETEC, and enteropathogenic $E$ coli (EPEC). ${ }^{8}$

The cyclic GMP signalling is a typical example of how microorganisms have been able to study the intestinal physiology of complex animals, to obtain information about guanylin, the natural ligand of the GC/cyclic GMP signalling, to genetically engineer agonists (EASTl) that activate this system, and to share this knowledge with other bacteria. Even more remarkable is the observation that some microorganisms were clever enough to synthesise a long lived superagonist of guanylin, as ST turned out to be 40 times more active than guanylin. Given the time needed by procaryotic organisms to assemble new genes, to maintain them in their limited genome, and to share this information with other microorganisms, it follows that bacteria probably learned about the cyclic GMP pathway thousands, if not millions of years before us.

\section{The zonulin system}

In recent years much has been discovered about the structure, function, and regulation of tight junctions. However, the precise mechanism(s) through which they operate are still incompletely understood. The discovery of Zot shed some light on the physiological mechanism of regulation of tight junction permeability. ${ }^{41}{ }^{42}$ We have recently reported that Zot possesses multiple domains that allow a dual function of the protein as a morphogenetic phage peptide for the Vibrio cholerae phage CTX $\phi$ and as an enterotoxin that modulates intestinal tight junctions. ${ }^{45}$

Zot enterotoxic action is mediated by a cascade of intracellular events that lead to a protein kinase C (PKC) dependent polymerisation of actin microfilaments strategically localised to regulate the paracellular pathway. ${ }^{42}$ The toxin exerts its effect by interacting with an intestinal surface receptor whose distribution coincides with the regional effect of Zot on intestinal permeability, ${ }^{62}$ and with the preferential F actin redistribution induced by Zot in the mature cells of the villi. $^{42}$ The expression of this receptor(s) seems to be up regulated during enterocyte differentiation. This hypothesis is supported by the observation that human intestinal epithelial CaCo 2 cells (that resemble the mature absorptive enteric cell of the villi), but not crypt-like T84 cells, express this receptor(s) on their surface..$^{6}$ The paucity of Zot binding in the crypt area may also reflect the fact that this region is already leaky compared to the more mature epithelium of the tip of the villi, ${ }^{64}$ and thus might not need to express a significant amount of a putative receptor(s) involved in tight junction regulation.

Taken together, these data showed that Zot regulates tight junctions in a rapid, reversible, and reproducible fashion, and probably activates intracellular signals which are operative during the physiological modulation of the paracellular pathway.

Based on this observation, it was postulated that Zot may mimic the effect of a functionally and immunologically related endogenous modulator of epithelial tight junctions. The combination of affinity purified anti-Zot antibodies and the Ussing chamber assay lead to the identification of zonulin, an intestinal Zot analogue. ${ }^{47}$ When zonulin was studied in a non-human primate model, it reversibly opened intestinal tight junctions. The discovery of the zonulin system has shed some light on the intricate pathophysiological regulation of intercellular tight junctions that, however, remains far from being completely addressed. It is conceivable that zonulin participates in the physiological regulation of intercellular tight junctions of the small intestine. Dysregulation of this conceptual zonulin model may contribute to disease states that involve disordered intercellular communication including developmental and intestinal disorders leading to autoimmune disease (coeliac disease and type 1 diabetes), tissue inflammation, malignant transformation, and metastasis. This same system can offer the opportunity of targeted, tissue specific delivery of macromolecules and drugs currently engineered by recombinant DNA techniques or that will become available through the human genome project. 


\section{REFERENCES}

1 Fasano A. Cellular microbiology: can we learn cell physiology from microorganisms? Am J Physiol 1999;276:C765-6.

2 Levine MM. Escherichia coli that cause diarrhea: enterotoxigenic, enterophatogenic, enteroinvasive, and enteroadherent. J Infect Dis 1987; 155:377-89

3 Rodighiero C, Aman AT, Kenny M, et al. Structural basis for the differential toxicity of cholera toxin and Escherichia coli heat-labile enterotoxin. J Biol Chem 1999;274:3962-9.

4 Okamoto K, Takahara M. Synthesis of Eschericia coli heat-stable enterotoxin Stp as a pre-pro form and role of the pro sequence in secretion. J Bacteriol 1990; 172:5260-5.

5 Yamanaka H, Nomura T, Fujii Y, Okamoto K. Need for TolC, an Escherichia coli outer membrane protein, in the secretion of heat-stable enterotoxin I across the outer membrane. Microb Pathog 1998;25: $111-20$.

6 Closs EJ, Ensseleit F, Koesling D, et al. Coexpression of inducible NO synthase and soluble guanylyl cyclase in colonic enterocytes: a pathophysiologic signaling pathway for the initiation of diarrhea by gram-negative bacteria? FASEB J 1998;12:1643-9.

7 Savarino SJ, Fasano A, Robertson DC, Levine MM. Enteroaggregative Escherichia coli elaborate a heat-stable enterotoxin demonstrable in an in vitro rabbit intestinal model. J Clin Invest 1991;87:1450-5.

8 Savarino SJ, McVeigh A, Watson J, et al. Enteroaggregative Escherichia coli heat-stable enterotoxin is not restricted to enteroaggregative $E$. coli. J Infect Dis 1996;173:1019-22.

9 Vila J, Gene A, Vargas M, et al. A case-control study of diarrhoea in children caused by Escherichia coli producing heat-stable enterotoxin (EAST 1). J Med Microbiol 1998;47:889-91.

10 Sulakvelidze A, Kreger A, Joseph A, et al. Production of enterotoxin by Yersinia bercovieri, a recently identified Yersinia enterocolitica-like species. Infect Immun 1999;67:968-71.

11 Fasano A, Hokama Y, Russell R, Morris JG Jr. Diarrhea in ciguatera fish poisoning: preliminary evaluation of pathophysiological mechanisms. Gastroenterology 1991;100:471-6.

12 Hughes S, Warhusst G, Turneberg LA, et al. Clostridium difficile toxin induced intestinal secretion in rabbit ileum in vitro. Gut 1983;24:94-8.

13 Guarino A, Berni Canani R, Pozio E, et al. Enterotoxin effect of stool supernatant of Cryptosporidium infected calves on human jejumun. Gastroenterology 1994;106:28-34.

14 Guarino A, Bisceglia M, Berni Canani R, et al. Enterotoxic effect of the vacuolating toxin produced by Helicobacter pylori in CaCo-2 cells. J Infect Dis 1998; 178:1373-8

15 Raimondi F, Kao JPY, Kaper JB, et al. Calcium-dependent intestinal chloride secretion by Vibrio parahaemolyticus thermostable direct hemolysin in a rabbit model. Gastroenterology 1995;109:381-6.

16 Izzo AA, Mascolo N, Capasso F. Nitric oxide as a modulator of intestinal water and electrolyte transport. Dig Dis Sci 1998;43:1605-20.

17 Wilson KT, Xie Y, Musch MW, Chang EB. Sodium nitroprusside stimulates anion secretion and inhibits $\mathrm{NaCl}$ absorption in rat colon. $J$ Pharmacol Exp Ther 1993;266:224-30.

18 Turvill JL, Mourad FH, Farthing MG. Proabsorptive and prosecretory roles of nitric oxide in cholera toxin induced secretion. Gut 1999;44:33-9.

19 Mascolo N, Izzo AA, Autore G, et al. Nitric oxide and castor oil-induced diarrhea. J Pharmacol Exp Ther 1994;268:291-5.

20 Dykhuizen RS, Masson J, McNight G, et al. Plasma nitrate concentration in infective gastroenteritis and inflammatory bowel diseases. Gut 1996;39:393-5

21 Fasano A, Noriega FR, Maneval RD Jr, et al. Shigella enterotoxin 1: an enterotoxin of Shigella flexneri 2 a active in rabbit small intestine. J Clin Invest 1995;95:2853-61.

22 Nataro JP, Seriwatana J, Fasano A, et al. Identification and cloning of a novel plasmid-encoded enterotoxin in enteroinvasive E.coli and Shigella. Infect Immun 1995;63:4721-8.

23 Noriega FR, Ming Liao F, Formal SB, et al. Prevalence of Shigella enterotoxin 1 (ShET1) among Shigella clinical isolates of diverse serotypes. J Infect Dis 1995;172:1408-10.

24 Rhee S, Wilson K, Gobert A, et al. The secretory effect of Shigella enterotoxin 1 (ShET1) is mediated by nitric oxide. Pediatr Res 2001:49:116A.

25 Popoff MR. Interaction between bacterial toxin and intestinal cells. Toxicon 1998;36:665-85.

26 Zitzer A, Zitzer O, Bhakdi S, Palmer M. Oligomerization of Vibrio cholerae cytolisin yields a pentameric pore and has a dual specificity for cholesterol and sphingolipids in the target membrane. J Biol Chem 1999;274: 1375-80

27 Kaper JB, O'Brien AD, eds. Escherichia coli O157:H7 and other Shiga toxin-producing E. coli strains. American Society for Microbiology, 1997.

28 Obrig TG, Louise CB, Lingwood CA, et al. Endothelial heterogeneity in Shiga toxin receptors and responses. J Biol Chem 1993:268: 15484-8.

29 Jacewicz MS, Acheson DWK, Binion DG, et al. Responses of human intestinal microvascular endothelial cells to Shiga toxins 1 and 2 and pathogenesis of hemorrhagic colitis. Infect Immun 1999;67:1439-44.

30 Boerlin P, McEwen SA, Boerlin-Petzold F, et al. Associations between virulence factors of Shiga toxin-producing Escherichia coli and disease in humans. J Clin Microbiol 1999;37:497-503

31 Nielsen MB, Odum N, Gerwien J, et al. Staphylococcus enterotoxin-A directly stimulated signal transduction and interferon-gamma production in psoriatic T-cell lines. Tissue Antigens 1998;52:530-8.

32 Guerrant RL, Steiner TS, Lima AM, Bobak DA. How intestinal bacteria cause disease. J Infect Dis 1999;179:S331-7.
33 Steiner TS, Lima AM, Nataro JP, Guerrant RL. Enteroaggregative Escherichia coli produce intestinal inflammation and growth impairment and cause interleukin-8 release from intestinal epithelial cells. J Infect Dis 1998;177:88-96

34 Borriello SP. Pathogenesis of Clostridium difficile infection. J Antimicrob Chemother 1998:41:13-19.

35 Aktories K. Bacterial toxins that target Rho proteins. J Clin Invest 1997:99:827-9

36 Narumija S, Ishizaki T, Watanabe N. Rho effectors and reorganization of actin cytoskeleton. FEBS Lett 1997;410:68-72.

37 Pothoulakis C. Pathogenesis of Clostridium difficile-associated diarrhoea. Eur J Gastroenterol Hepatol 1996:8:1041-7.

38 Falbo V, Pace T, Picci L, et al. Isolation and nucleotide sequence of the gene encoding cytotoxic necrotizin factor 1 of Escherichia coli. Infect Immun 1993;61:4909-14

39 Schmidt G, Sehr P, Wilm M, et al. Deamination of Gln63 of Rho induced by Escherichia coli cytotoxic necrotizing factor 1. Nature 1997:387:725-9.

40 Gerhard R, Schmidt G, Hofmann F, Aktories K. Activation of Rho GTPases by Escherichia coli cytotoxic necrotizing factor 1 increases intestinal permeability in CaCo2 cells. Infect Immun 1998;66:5125-31.

41 Fasano A, Baudry B, Pumplin DW, et al. Vibrio cholerae produces a second enterotoxin, which affects intestinal tight junctions. Proc Natl Acad Sci U S A 1991;88:5242-6.

42 Fasano A, Fiorentini C, Donelli G, et al. Zonula occludens toxin (ZOT) modulates tight junctinos through protein kinase $\mathrm{C}$-dependent actin reorganization, in vitro. J Clin Invest 1995;96:710-20.

43 Waldor MK, Mekalanos JJ. Lysogenic conversion by a filamentous phage encoding cholera toxin. Science 1996;272:1910-14

44 Fasano A. Cellular microbiology: how enteric pathogens socialize with their intestinal host. J Pediatr Gastroenterol Nutr 1998;26:520-32.

45 Uzzau S, Cappuccinelli P, Fasano A. Expression of Vibrio cholerae zonula occludens toxin and analysis of its subcellular localization. Microb Pathogen 1999;27:377-85.

46 Di Pierro M, Lu R, Uzzau S, et al. Zonula occludens toxin structure-function analysis: identification of the fragment biologically active on tight junctions and of the zonulin receptor binding domain. $J$ Biol Chem 2001;216:19160-5.

47 Wang W, Uzzau S, Goldblum SE, Fasano A. Human zonulin, a potential modulator of intestinal tight junctions. J Cell Sci 2000;1 13:4435-40.

48 Navarro-Garcia F, Sears C, Eslava C, et al. Cytoskeletal effects induced by Pet, the serine protease enterotoxin of enteroaggregative Escherichia coli. Infect Immun 1999:67:2184-92.

49 Wu S, Lim K-C, Huang J, et al. Bacteroides fragilis enterotoxin cleaves the zonula adherens protein E-cadherin. Proc Natl Acad Sci U S A 1998;95: 14979-84.

50 Fabbri A, Falzano L, Frank C, et al. Vibrio parahaemolyticus thermostable direct hemolysin modulates cytoskeletal organization and calcium homeostasis in intestinal cultured cells. Infect Immun 1999;67: 1139-48.

51 Faruque SM, Asadulghani, Saha MN, et al. Analysis of clinical and environmental strains of nontoxigenic Vibrio cholerae for susceptibility to CTXPhi: molecular basis for origination of new strains with epidemic potential. Infect Immun 1998:66:5819-25.

52 Kimsey HH, Nair GB, Ghosh A, Waldor MK. Diverse CTX $\phi$ s and evolution of new pathogenic Vibrio cholerae. Lancet 1998;352:457-8.

53 Maurelli AT, Fernandez RE, Block CA, et al. "Black holes" and bacterial pathogenicity: a large genomic deletion that enhances the virulence of Shigella spp. and enteroinvasive Escherichia coli. Proc Natl Acad Sci U S A 1998;95:3943-8.

54 Frantz JC, Jaso-Friedman L, Robertson DC. Binding of Escherichia coli heat-stable enterotoxin to rat intestinal cells and brush border membranes. Infect Immun 1984;43:622-30.

55 Giannella RA, Luttrell M, Thompson M. Binding of Escherichia coli heat-stable enterotoxin to receptors on rat intestinal cells. Am J Physiol 1983;245:G492-8

56 Goldberg MB, Sansonetti PJ. Shigella subversion of the cellular cytoskeleton: a strategy for epithelial colonization. Infect Immun 1993:61:4941-6.

57 Giannella RA. Escherichia coli heat-stable enterotoxins, guanylins, and their receptors: what are they and what do they do? J Lab Clin Med1995;125:173-81.

58 Schulz S, Green CK, Yuen PS, Garbers DL. Guanylyl cyclase is a heat-stable enterotoxin receptor. Cell 1990;63:941-8.

59 Schulz S, Singh S, Bellet RA, et al. The primary structure of a plasma membrane guanylate cyclase demonstrates diversity within this new receptor family. Cell 1989;58:1 155-62.

60 Currie MG, Fok KF, Kato J, et al. Guanylin: an endogenous activator of intestinal guanylate cyclase. Proc Natl Acad Sci U S A 1992;89:947-51.

61 Savarino SJ, Fasano A, Watson J, et al. Enteroaggregative Escherichia coli heat-stable enterotoxin 1 represents another subfamily of $E$. coli heat-stable toxin. Proc Natl Acad Sci U S A 1993;90:3093-7.

62 Fasano A, Uzzau S, Fiore C, Margaretten K. The enterotoxic effect of zonula occludens toxin (Zot) on rabbit small intestine involves the paracellular pathway, Gastroenterology 1997;1 12:839-46.

63 Uzzau S, Lu R, Wang W, et al. Purification and preliminary characterization of the zonula occludens toxin receptor from human $(\mathrm{CaCo})$ and murine (IEC6) intestinal cell lines. FEMS Microbiol Lett $2001 ; 1: 1-5$

64 Marcial MA, Carlson SL, Madara JL. Partitioning of paracellular conductance along the ileal crypt-villus axis: a hypothesis based on structural analysis with detailed consideration of tight junction structure-function relationships. J Membr Biol 1984;80:59-64 